\title{
ANALYTIC REPRESENTATION OF GENERALIZED TEMPERED DISTRIBUTIONS OF EXPONENTIAL GROWTH BY WAVELETS
}

\author{
By \\ Byung Keun SoHN and Dae Hyeon PaHK
}

\begin{abstract}
The analytic representation of the generalized tempered distributions of $e^{M(k x)}$-growth with restricted order, $\mathscr{K}_{M}^{r^{\prime}}(R)$, is given in terms of series of analytic wavelets. These series converge uniformly on compact subsets of the upper and lower half planes.
\end{abstract}

\section{Introduction}

The analytic representation of functions or distributions on the real line $R$ is usually given by a Cauchy type formula, but in some cases may also be given by an orthogonal series. It is well-known that trigonometric series may be used for the analytic representation of periodic functions and distributions. Also, Hermite series and Legendre polynomials can be used for the representation of nonperiodic functions and functions with compact support, respectively. Recently a new category of orthogonal systems has been introduced in [1]. These systems are composed of wavelets, i.e., orthogonal functions on $R$ consisting of dilations and translations of a fixed function. G. G. Walter has found an expansion in orthogonal wavelets and pointwise convergence of that expansion from $L^{2}(R)$ to the tempered distributions with restricted order of derivative, $\mathscr{S}_{r}^{\prime}(R)$, in [6] and [8] and has showed an analytic representation of $\mathscr{S}_{r}^{\prime}(R)$ in terms of series of analytic wavelets in [7]. These two results were extended by us to the case of the tempered distributions of exponential growth with restricted order in [3], [5]. Also, we have found the wavelet expansion of the tempered distributions of $e^{M(k x)}$-growth

2000 Mathematics Subject Classification: 41A15, 46F20.

Key words and phrases: generalized tempered distribution, wavelet, analytic representation.

The first author was supported by Inje Research and Scholarship Foundation 2000-20001 and the second author was supported by KRF 99-005-D00009.

Received June 5, 2001.

Revised July 8, 2002. 
with restricted order and the pointwise convergence of the wavelet expansion of $\mathscr{K}_{M}^{r \prime}(R)$ in [4].

In this paper, we will present an analytic representation of $\mathscr{K}_{M}^{r \prime}(R)$ in terms of series of analytic wavelets. These series converge uniformly on compact subsets of the upper and lower half planes.

\section{The Generalized Tempered Distributions Space $\mathscr{K}_{M}^{\prime}(R)$}

Let $\mu(\xi)(0 \leq \xi \leq \infty)$ denote a continuous increasing function such that $\mu(0)=0, \mu(\infty)=\infty$. For $x \geq 0$, we define

$$
M(x)=\int_{0}^{x} \mu(\xi) d \xi
$$

The function $M(x)$ is an increasing, convex and continuous function with $M(0)=0, M(\infty)=\infty$ and satisfies the fundamental convexity inequality $M\left(x_{1}\right)+M\left(x_{2}\right) \leq M\left(x_{1}+x_{2}\right)$. Further we define $M(x)$ for negative $x$ by means of the equality $M(-x)=M(x)$. Note that since the derivative $\mu(x)$ of $M(x)$ is unbounded in $R$, the function $M(x)$ will grow faster than any linear function as $|x| \rightarrow \infty$. Now we list some properties of $M(x)$ which will be frequently used in this paper.

$$
\begin{gathered}
M(x)+M(y) \leq M(x+y) \text { for all } x, y \geq 0 \\
M(x+y) \leq M(2 x)+M(2 y) \text { for all } x, y \geq 0
\end{gathered}
$$

Using the function $M(x)$ we define the space $\mathscr{K}_{M}(R)$ as the space of all functions $\phi \in C^{\infty}(R)$ such that

$$
v_{k}(\phi)=\sup _{x \in R, \alpha \leq k} e^{M(k x)}\left|D^{\alpha} \phi(x)\right|<\infty, \quad k=1,2, \ldots,
$$

where $D^{\alpha}=d^{\alpha} / d x^{\alpha}$. The topology in $\mathscr{K}_{M}(R)$ is defined by the family of the seminorms $v_{k}$. Then $\mathscr{K}_{M}(R)$ becomes a Fréchet space and the embeddings $\mathscr{D} \hookrightarrow$ $\mathscr{K}_{M} \hookrightarrow \mathscr{S} \hookrightarrow \mathscr{E}$ are continuous; here $\mathscr{E}$ denotes the space of all $C^{\infty}$-functions, $\mathscr{S}$ the space of the tempered distributions of polynomial growth and $\mathscr{D}$ the space of $C^{\infty}$-functions with compact supports. By $\mathscr{K}_{M}^{\prime}(R)$, we mean the space of continuous linear functionals on $\mathscr{K}_{M}(R)$. Pahk characterized the distributions in $\mathscr{K}_{M}^{\prime}(R)$ by the growth at infinity [2, Theorem 2.3]; a distribution $T \in \mathscr{D}^{\prime}$ is in $\mathscr{K}_{M}^{\prime}(R)$ if and only if there exist positive integers $\alpha, k_{0}$ and a bounded continuous function $f(x)$ on $R$ such that

$$
T=D^{\alpha}\left[e^{M\left(k_{0} x\right)} f(x)\right]
$$


DeFINITION 1. For a natural number $r$, we denote by $\mathscr{K}_{M}^{r}(R)$ the space of all functions $\phi \in C^{r}(R)$ such that

$$
v_{k}^{r}(\phi)=\sup _{x \in R, \alpha \leq r} e^{M(k x)}\left|D^{\alpha} \phi(x)\right|<\infty, \quad k=1,2,3, \ldots
$$

The topology of $\mathscr{K}_{M}^{r}(R)$ is defined by the family of semi-norms $\left\{v_{k}^{r}\right\}_{k=1,2, \ldots}$. By $\mathscr{K}_{M}^{r \prime}(R)$, we mean the space of continuous linear functionals on $\mathscr{K}_{M}^{r}(R)$. Each $S \in \mathscr{K}_{M}^{r \prime}(R)$ is characterized by

$$
S=D^{r}\left[e^{M\left(k_{0} x\right)} f(x)\right]
$$

where $f(x)$ is a bounded continuous function on $R$ and $r, k_{0} \in N$, the set of natural numbers, by the same method of the above $\mathscr{K}_{M}^{\prime}$-case in [2, Theorem 2.3]. Similarly, we can define

$$
\mathscr{S}_{r}(R)=\left\{\theta(t) \in C^{r}(R) ;\left|D^{k} \theta(t)\right| \leq C_{p k}(1+|t|)^{-p}, p \in N, k=0,1, \ldots, r\right\}
$$

and its dual $\mathscr{S}_{r}^{\prime}(R)$. For further details, we refer to [2].

\section{Multiresolution Analysis of $L^{2}(R)$ Associated with $\phi \in \mathscr{K}_{M}^{r}(R)$}

Let $\phi \in \mathscr{K}_{M}^{r}(R)$. In order for it to qualify as a scaling function, there must be associated with $\phi$ a multiresolution analysis of $L^{2}(R)$, i.e., a nested sequence of closed subspaces $\left\{V_{m}\right\}_{m \in Z}$ for the set of integers $Z$ such that

(i) $\{\phi(\cdot-n)\}$ is an orthonormal basis of $V_{0}$,

(ii) $\cdots \subset V_{-1} \subset V_{0} \subset V_{1} \subset \cdots \subset L^{2}(R)$,

(iii) $f(\cdot) \in V_{m} \Leftrightarrow f(2 \cdot) \in V_{m+1}$,

(iv) $\bigcap_{m} V_{m}=\{0\}, \overline{\bigcap_{m} V_{m}}=L^{2}(R)$.

Then $\phi$ has an expansion

$$
\phi(t)=\sum_{n} c_{n} \sqrt{2} \phi(2 t-n), \quad\left\{c_{n}\right\} \in l^{2}, t \in R
$$

where $l^{2}=\left\{\left\{c_{n}\right\} ; \sum_{n}\left|c_{n}\right|^{2}<\infty\right\}$. Once we have the scaling function $\phi \in \mathscr{K}_{M}^{r}(R)$, we can obtain a mother wavelet $\psi$ such that $\{\psi(t-n)\}$ is an orthogonal basis of the space $W_{0}$, given by the orthogonal complement of $V_{0}$ in $V_{1}$. Also, $\psi$ has an expansion

$$
\psi(t)=\sum_{n} d_{n} \sqrt{2} \phi(2 t-n), \quad\left\{d_{n}\right\} \in l^{2}
$$


for $d_{n}$ corresponding to $c_{n}$ in (5). We will adopt the construction of a mother wavelet defined by $d_{n}=(-1)^{n} \overline{c_{1-n}}$. If such a $\psi(t)$ can be found, then $\psi_{m n}(t)=$ $2^{m / 2} \psi\left(2^{m} t-n\right)$ is an orthogonal basis of $W_{m}$ which is the orthogonal complement of $V_{m}$ in $V_{m+1}$.

EXAMPLE. In [1], Corollary 5.5.3 states that it is impossible that $\psi$ has exponential decay and that $\psi \in C^{\infty}$, with all derivatives bounded, unless $\psi=0$. Hence there is no mother wavelet $\psi \in \mathscr{K}_{M}(R)$. So we will restrict our attention to $\mathscr{K}_{M}^{r}(R)$. Daubechies' compactly supported wavelets are examples of $\mathscr{K}_{M}^{r}(R)$, but Battle-Lemarié's wavelets (in the page 152 of [1]) are not $\mathscr{K}_{M}^{r}(R)$ wavelets even if they have exponential decay and smoothness.

The reproducing kernel of $V_{0}$ is given by

$$
q(x, t)=\sum_{n} \overline{\phi(x-n)} \phi(t-n)
$$

where $\phi(x)$ is the scaling function. The series and its derivatives with respect to $t$ of order $\leq r$ converge uniformly on $x \in R$ because of the regularity of $\phi \in \mathscr{K}_{M}^{r}(R)$, i.e.,

$$
\left|\phi^{(\alpha)}(x)\right| \leq C_{\alpha k} e^{-M(k x)}, \quad \alpha=0,1, \ldots, r ; k=1,2, \ldots
$$

The reproducing kernel for $V_{m}$ is given by

$$
q_{m}(x, t)=2^{m} q\left(2^{m} x, 2^{m} t\right) .
$$

Similarly, we can define the reproducing kernel $r_{m}(x, t)$ for $W_{m}$ by

$$
r_{m}(x, t)=2^{m} \sum_{n} \overline{\psi\left(2^{m} x-n\right)} \psi\left(2^{m} t-n\right)
$$

where $\psi(t)$ is the mother wavelet.

The sequence $\left\{q_{m}(x, t)\right\}$ is a delta sequence in $\mathscr{S}_{r}^{\prime}(R) \subset \mathscr{K}_{M}^{r \prime}(R)$, i.e., $q_{m}(x, t) \rightarrow \delta(x-t)$. This follows from the fact that

$$
\int_{-\infty}^{\infty} q_{m}(x, t) \theta(t) d t \rightarrow \theta(x) \quad \text { as } m \rightarrow \infty
$$

for each $\theta \in \mathscr{K}_{M}^{r}(R) \subset \mathscr{S}_{r}(R)$, where the convergence is in the $L^{2}$-sense. These kernels have a number of interesting properties, some of which come out of the wavelet moment theorem. Since $\mathscr{K}_{M}^{r}(R) \subset \mathscr{S}_{r}(R)$, we have by [1], 
LEMMA 2. Let $\psi \in \mathscr{K}_{M}^{r}(R)$ with $\psi_{m n}(x)=2^{m / 2} \psi\left(2^{m} x-n\right)$ an orthogonal system in $L^{2}(R)$. Then

$$
\int_{-\infty}^{\infty} x^{k} \psi(x) d x=0, \quad k=0,1, \ldots, r .
$$

Definition 3. We define the spaces $T_{0}$ and $U_{0}$ by $T_{0}=\{f ; f(t)=$ $\sum_{n} a_{n} \phi(t-n)$ for some sequence of complex numbers with $a_{n}=\mathcal{O}\left(e^{M\left(k_{1} n\right)}\right)$ for some $\left.k_{1} \in N\right\}$ and $U_{0}=\left\{g ; g(t)=\sum_{n} a_{n} \psi(t-n)\right.$ for some sequence of complex numbers with $a_{n}=\mathcal{O}\left(e^{M\left(k_{1} n\right)}\right)$ for some $\left.k_{1} \in N\right\}$. We denote by $T_{m}$ and $U_{m}$ their corresponding dilation spaces, i.e., $f \in T_{0} \Leftrightarrow f\left(2^{m} t\right) \in T_{m}$ and $g \in U_{0} \Leftrightarrow g\left(2^{m} t\right) \in$ $U_{m}$.

We may expect that a multiresolution analysis of $\mathscr{K}_{M}^{r \prime}(R)$ exists, namely,

$$
\cdots \subset T_{-m} \cdots \subset T_{-1} \subset T_{0} \subset T_{1} \cdots \subset T_{m} \subset \cdots \subset \mathscr{K}_{M}^{r \prime}(R)
$$

and

$$
\overline{\bigcup_{m} T_{m}}=\mathscr{K}_{M}^{r \prime}(R)
$$

where the closure is in the topology of $\mathscr{K}_{M}^{r \prime}(R)$.

Now in [3], we have found the expansion in orthogonal wavelets from $L^{2}(R)$ to $\mathscr{K}_{M}^{r \prime}(R)$.

THEOREM 4. Let the scaling function $\phi \in \mathscr{K}_{M}^{r}(R)$ satisfy the dilation equation (5) with $c_{k}=\mathcal{O}\left(e^{-M(l k)}\right)$ for all $l \in N$, and have an associated multiresolution analysis in $L^{2}(R)$; let $\psi \in \mathscr{K}_{M}^{r}(R)$ be the mother wavelet given in (6). Then there exists a multiresolution analysis (8) of closed dilation subspaces $\left\{T_{m}\right\}$ whose union is dense in $\mathscr{K}_{M}^{r \prime}(R)$; the closed subspace $U_{m}$ in Definition 3 is a complementary subspace of $T_{m}$ in $T_{m+1}$ and

$$
T_{m}=U_{0} \oplus U_{1} \oplus \cdots \oplus U_{m} \oplus T_{0},
$$

where $\oplus$ denotes the nonorthogonal direct sum.

\section{Analytic Representation of Distributions of $\mathscr{K}_{M}^{r \prime}$ by Wavelets}

A quasi-positive delta sequence is a sequence $\left\{\delta_{m}(\cdot, y)\right\}$ of functions in $L^{1}(R)$ with a parameter $y \in R$ which satisfies the following:

(a) there is a $C>0$ such that

$$
\int_{-\infty}^{\infty}\left|\delta_{m}(x, y)\right| d x \leq C, \quad y \in R, m \in N
$$


(b) there is a $c>0$ such that

$$
\int_{y-c}^{y+c} \delta_{m}(x, y) d x \rightarrow 1
$$

uniformly on compact subsets of $R$, as $m \rightarrow \infty$;

(c) for each $\gamma>0$,

$$
\sup _{|x-y| \leq \gamma}\left|\delta_{m}(x, y)\right| \rightarrow 0 \quad \text { as } m \rightarrow \infty .
$$

Then since $\mathscr{K}_{M}^{r}(R) \subset \mathscr{S}_{r}(R)$, we have the following important lemmas as in [8]:

Lemma 5. Let $\left\{\delta_{m}(x, y)\right\}$ be a quasi-positive delta sequence and let $f \in L^{1}(R)$ be continuous on $(a, b)$. Then

$$
f_{m}(y)=\int_{-\infty}^{\infty} \delta_{m}(x, y) f(x) d x \rightarrow f(y) \quad \text { as } m \rightarrow \infty
$$

uniformly on compact subsets of $(a, b)$.

LEMMA 6. If the scaling function $\phi \in \mathscr{K}_{M}^{r}(R)$, then the reproducing kernel $q_{m}(x, y)$ and $K_{m}(x, t)=\frac{(x-t)}{\alpha !} \frac{\partial^{\alpha}}{\partial t^{\alpha}} q_{m}(x, t)$ for $\alpha \in N, 0 \leq \alpha \leq r$, are quasi-positive delta sequences on $R$.

In order to represent an element of $\mathscr{K}_{M}^{r \prime}(R)$ by series of analytic wavelets, we impose conditions on the scaling function $\phi$ again. Since $\mathscr{K}_{M}^{r}(R) \subset L^{2}(R)$, an analytic representation of $\phi$ is given by

$$
\phi^{ \pm}(z)=\frac{1}{2 \pi i} \int_{-\infty}^{\infty} \frac{\phi(x)}{x-z} d x, \quad \operatorname{Im} z \gtrless 0,
$$

where $\phi^{ \pm}$are analytic in the upper half-plane and the lower half-plane, respectively. An analytic representation of the mother wavelet is also given by

$$
\psi^{ \pm}(z)=\frac{1}{2 \pi i} \int_{-\infty}^{\infty} \frac{\psi(x)}{x-z} d x, \quad \operatorname{Im} z \gtrless 0,
$$

and the analytic wavelets $\psi_{m n}^{ \pm}$are obtained by dilation and translation of $\psi^{ \pm}$. Now, we define $T_{0}^{ \pm}=\left\{f(z)=\sum_{n} a_{n} \phi^{ \pm}(z-n) ; a_{n}=\mathcal{O}\left(c^{l_{0} M(n)}\right)\right.$ for some $\left.l_{0} \in N\right\}$ and we denote by the subspaces $T_{m}^{ \pm}$of $T_{0}^{ \pm}$the corresponding dilation spaces. Then the spaces $T_{m}^{+}$and $T_{m}^{-}$are composed of analytic functions in the upper and the lower half-planes, respectively, whose boundary functions are continuous 
functions of $e^{M(x)}$-growth. Since $\overline{\bigcup T_{m}}=\mathscr{K}_{M}^{r \prime}(R)$, we might expect to obtain an analytic representation of $f \in \mathscr{K}_{M}^{r \prime}(R)$ in terms of wavelets,

$$
f^{+}(z)=\sum_{n=-\infty}^{\infty} a_{n} \phi^{+}(z-n)+\sum_{m=0}^{\infty} \sum_{n=-\infty}^{\infty} b_{m n} 2^{m / 2} \psi^{+}\left(2^{m} z-n\right)
$$

where the first series may not converge. Since an analytic representation is a continuous map from $\mathscr{K}_{M}^{r \prime}(R)$ to a corresponding space of analytic functions and $f_{m}(x)=\left(f, q_{m}(x, t)\right) \rightarrow f(x)=D^{r} F(x)$ in $\mathscr{K}_{M}^{r \prime}(R)$ for a continuous function of $e^{M(x)}$-growth $F(x)$ [cf. (4)] by Lemmas 5 and $6, f_{m}^{+}(z) \rightarrow f^{+}(z)$ uniformly on bounded subsets of the upper half-plane. Moreover, $f^{+}(z)=D_{z}^{r} F^{+}(z)$, where $F^{+}(z)$ is an analytic representation of $F(z)$, and is given by

$$
F^{+}(z)=\frac{1}{2 \pi i} \int_{-\infty}^{\infty} \frac{F(x)}{x-z} e^{-M(k x)} e^{M(k z)} d x,
$$

for a sufficiently large $k$ such that $F(x) e^{-M(k x)} \in L^{2}(R)$. Here for $z \in C$, we define $e^{M(z)}$ as $e^{M(|z|)}$.

We may express $f_{m}$ as

$$
f_{m}=f_{0}+f_{m}-f_{0}=f_{0}+\sum_{k=0}^{m-1} \sum_{n=-\infty}^{\infty} b_{k n} \psi_{k n},
$$

and if the inner sum converges,

$$
f_{m}^{+}(z)-f_{0}^{+}(z)=\sum_{k=0}^{m-1} \sum_{n=-\infty}^{\infty} b_{k n} \psi_{k n}^{+}(z)+g_{m}(z)
$$

where $g_{m}(z)$ is an entire function.

LEMMA 7. Let $\psi \in \mathscr{K}_{M}^{r}(R)$ and $b_{n}=\mathcal{O}\left(e^{M(k n)-\varepsilon}\right)$ for any $k \in N$ and some $\varepsilon>0$. Then

$$
\sum_{n=-\infty}^{\infty} b_{n} \psi^{+}(z-n)
$$

converges uniformly on compact subsets of the upper half-plane.

ProOF. The proof is based on the moment property, Lemma 2,

$$
\int_{-\infty}^{\infty} x^{l} \psi(x) d x=0, \quad l=0,1, \ldots, r .
$$

Hence, for any $k \in N$ and a natural number $p \leq r+1$, 


$$
\begin{aligned}
e^{M(k z)} \psi^{+}(z)= & \frac{1}{2 \pi i} \int_{-\infty}^{\infty} \frac{e^{M(k z)}}{z^{p}} \cdot \frac{z^{p}}{x-z} \psi(x) d x \\
= & \frac{1}{2 \pi i} \int_{-\infty}^{\infty} \frac{e^{M(k z)}}{z^{p}} \cdot \frac{z^{p}-x^{p}}{x-z} \psi(x) d x \\
& +\frac{1}{2 \pi i} \int_{-\infty}^{\infty} \frac{e^{M(k z)}}{z^{p}} \cdot \frac{x^{p}}{x-z} \psi(x) d x \\
= & -\frac{1}{2 \pi i} \int_{-\infty}^{\infty} \frac{e^{M(k z)}}{z^{p}} \cdot\left(x^{p-1}+z x^{p-2}+\cdots+z^{p-2} x+z^{p-1}\right) \psi(x) d x \\
& +\frac{1}{2 \pi i} \int_{-\infty}^{\infty} \frac{e^{M(k z)}}{z^{p}} \cdot \frac{x^{p}}{x-z} \psi(x) d x \\
= & \frac{1}{2 \pi i} \int_{-\infty}^{\infty} \frac{e^{M(k z)}}{z^{p}} \cdot \frac{x^{p}}{x-z} \psi(x) d x
\end{aligned}
$$

holds. By the growth condition of $\psi \in \mathscr{K}_{M}^{r}(R),\left|e^{M(k z)} \psi^{+}(z)\right|$ is uniformly bounded on compact subsets of the half-plane $\operatorname{Im} z \geq \varepsilon>0$ for any $k \in N$ and a natural number $p \leq r+1$. Hence, the preceding fact holds for any $k \in N$ and any $p \leq r+1$. Thus the conclusion follows.

THEOREM 8. For natural numbers $s, r$ with $s<r$, let $f \in \mathscr{K}_{M}^{s \prime}(R), \phi, \psi \in$ $K_{M}^{r}(R)$ and let $b_{m n}=\left\langle f, \psi_{m n}\right\rangle, m=0,1,2, \ldots ; n=0, \pm 1, \pm 2, \ldots$ be the wavelet coefficients of $f$. Then an analytic representation of $f$ is given by

$$
f^{+}(z)=f_{0}^{+}(z)+\sum_{m=0}^{\infty} \sum_{n=-\infty}^{\infty} b_{m n} \psi_{m n}^{+}(z)
$$

where the series converges uniformly on compact subsets of the half-plane $\operatorname{Im} z \geq 1$ and $f_{0}^{+}(z)$ is an analytic representation of $f_{0}$, the projection of $f$ on $T_{0}$.

ProOF. First, we will estimate $\left|b_{m n}\right|$. Each $f \in \mathscr{K}_{M}^{s \prime}(R)$ is characterized by

$$
f=D^{s}\left[e^{M\left(k_{0} x\right)} \mu\right]
$$

for some integer $k_{0}$ and finite measure $\mu$ on $R$. Each $\psi \in \mathscr{K}_{M}^{r}(R)$ satisfies

$$
\left|\psi^{(l)}(x)\right| \leq C_{j} e^{-M(j x)}, \quad l=1,2, \ldots, r ; j \geq 0 .
$$

If we use integration by parts $s$-times, we have, for $m>1$, 


$$
\begin{aligned}
\left|b_{m n}\right| & \leq \int_{-\infty}^{\infty}\left|D^{s}\left[e^{M\left(k_{0} x\right)}\right] \psi_{m n}(x)\right| d|\mu| \leq \int_{-\infty}^{\infty} e^{M\left(k_{0} x\right)}\left|\psi_{m n}^{(s)}(x)\right| d|\mu| \\
& \leq \int_{-\infty}^{\infty} e^{M\left(k_{0} x\right)} c_{k_{0}} 2^{m / 2+s m} e^{-M\left(k_{0}\left(2^{m} x-n\right)\right)} d|\mu| \\
& \leq \int_{-\infty}^{\infty} e^{M\left(2 k_{0}\left(x-n 2^{-m}\right)\right)} e^{M\left(2 k_{0} n 2^{-m}\right)} c_{k_{0}} 2^{m / 2+s m} e^{-M\left(2^{m} k_{0}\left(x-n 2^{-m}\right)\right)} d|\mu| \\
& \leq c_{k_{0}}^{\prime} 2^{m / 2+s m} e^{M\left(2 k_{0} n 2^{-m}\right)}
\end{aligned}
$$

By the fact in the proof of Lemma 7, on every compact subset $K$ of the halfplane $\operatorname{Im} z \geq 1$, there exists a constant $c$ such as $\left|\psi^{+}(z)\right| \leq c e^{-M(k z)}$ for any $k \in N$. Hence if we take $k$ sufficiently large with $k>\sup \left\{k_{0}, \frac{3}{2}+s\right\}$, then for $z \in K$,

$$
\begin{aligned}
\sum_{m=0}^{\infty} & \sum_{n=-\infty}^{\infty}\left|b_{m n} \psi_{m n}^{+}(z)\right| \\
\leq & \sum_{m=0}^{\infty} \sum_{n=-\infty}^{\infty} c_{k_{0}}^{\prime} e^{(1 / 2+s) m} e^{M\left(2 k_{0} n 2^{-m}\right)} c 2^{m / 2} e^{-M\left(k\left(2^{m} z-n\right)\right)} \\
\leq & \sum_{m=0}^{\infty} \sum_{n=-\infty}^{\infty} c c_{k_{0}}^{\prime} e^{(3 / 2+s) m} e^{M\left(2 k_{0} n 2^{-m}\right)} e^{-M\left(k 2^{m}\left(n 2^{-m}-R e z-i I m z\right)\right)} 2^{-m / 2} \\
\leq & \sum_{m=0}^{\infty} \sum_{n=-\infty}^{\infty} c c_{k_{0}}^{\prime} e^{(3 / 2+s) m} e^{M\left(2 k_{0} n 2^{-m}\right)} \\
& \times e^{-M\left(k 2^{m}\left(n 2^{-m}-R e z\right)\right)} e^{-M\left(2^{m-1} k\right)} 2^{-m / 2} \\
\leq & \left\{\sum_{m=0}^{2} \sum_{n=-\infty}^{\infty}+\sum_{m=3}^{\infty} \sum_{n=-\infty}^{\infty}\right\} c c_{k_{0}}^{\prime} e^{(3 / 2+s) m} e^{M\left(2 k_{0} n 2^{-m}\right)} \\
& \times e^{-M\left(k 2^{m}\left(n 2^{-m}-\operatorname{Re} z\right)\right)} e^{-M\left(2^{m-1} k\right)} 2^{-m / 2} \\
\leq & \sum_{m=3}^{\infty} C_{k_{0}, z} 2^{-m / 2}<\infty
\end{aligned}
$$

where we use the properties (1), (2) and the inequality $\sqrt{2}|z| \geq|\operatorname{Re} z|+|\operatorname{Im} z| \geq$ $|\operatorname{Re} z|+1$ for $|\operatorname{Im} z| \geq 1$. Hence the series $\sum_{m=0}^{\infty} \sum_{n=-\infty}^{\infty} b_{m n} \psi_{m n}^{+}(z)$ converges uniformly on compact subsets of the half-plane $\operatorname{Im} z \geq 1$.

Now, by taking the limit in (9) as $m \rightarrow \infty$, we have

$$
f^{+}(z)=f_{0}^{+}(z)+\sum_{k=0}^{\infty} \sum_{n=-\infty}^{\infty} b_{k n} \psi_{k n}^{+}(z)+g_{\infty}(z)
$$

where $g_{\infty}(z)=\lim _{m \rightarrow \infty} g_{m}(z)$ is an entire function. Since an analytic representation plus an entire function is an analytic representation, we can drop $g_{\infty}$ in (9).

REMARK. We have only worked out the convergence for $f^{+}$but proof 
for $f^{-}$is parallel. Then by the same method as in the proof of Theorem 8 , an analytic representation of $f$ is given by

$$
f^{-}(z)=f_{0}^{-}(z)+\sum_{m=0}^{\infty} \sum_{n=-\infty}^{\infty} b_{m n} \psi_{m n}^{-}(z)
$$

where the series converges uniformly on compact subsets of the half-plane $\operatorname{Im} z \leq-1$ and $f_{0}^{-}(z)$ is an analytic representation of $f_{0}$, the projection of $f$ on $T_{0}$.

\title{
References
}

[1] I. Daubechies, Ten Lectures on Wavelets, SIAM, CMBS 61, Philadelphia, 1992.

[2] Dae Hyoen Pahk, Structure theorem and Fourier transform for distributions with restricted growth, Kyungpook J. Math., 23(2) (1983), 129-146.

[3] Byung Keun Sohn and Dae Hyeon Pahk, Wavelets in the generalized tempered distributions, Tsukuba J. Math., 23(3) (1999), 529-538.

[4] Byung Keun Sohn and Dae Hyeon Pahk, Pointwise Convergence of Wavelets Expansion of $\mathscr{K}_{p}^{\prime}$, Bull. Korean Math. Soc., 38(1) (2001), 81-91.

[5] Byung Keun Sohn and Dae Hyeon Pahk, Analytic Representation of Generalized Tempered Distributions by Wavelets, Publ. RIMS, Kyoto Univ., 37(2) (2001), 141-149.

[6] G. G. Walter, Wavelets and generalized functions, Wavelets-a Tutorial, C. Chui (Ed.), Academic Press, Boston, 1992.

[7] G. G. Walter, Analytic representations with wavelet expansions, Complex Variables Theory Appl., 26(3) (1994), 235-243.

[ 8 ] G. G. Walter, Pointwise convergence of wavelet expansions, J. Approx. Theory, 80(1) (1995), 108-118.

\author{
Byung Keun Sohn \\ Department of Mathematics \\ Inje University \\ Kyungnam Kimhae 621-749, (South)KOREA \\ and \\ Dae Hyeon Pahk \\ Department of Mathematics \\ Yonsei University \\ Seoul 120-749, (South)KOREA
}

